\title{
Does rhythm matter in acute heart failure? An insight from the British Society for Heart Failure National Audit
}

\author{
Simon G. Anderson ${ }^{1,2,3} \cdot$ Ahmad Shoaib $^{4}$. Phyo Kyaw Myint ${ }^{5}$. John G. Cleland ${ }^{6,7}$. Suzanna M. Hardman ${ }^{8}$. \\ Theresa A. McDonagh ${ }^{9} \cdot$ Henry Dargie $^{10} \cdot$ Bernard Keavney $^{1} \cdot$ Clifford J. Garratt $^{1} \cdot$ Mamas A. Mamas $^{4}$
}

Received: 30 December 2018 / Accepted: 19 March 2019 / Published online: 8 April 2019

(c) The Author(s) 2019

\begin{abstract}
Background Atrial fibrillation (AF) is the most common sustained arrhythmia in patients with acute heart failure (AHF). The presence of AF is associated with adverse prognosis in patients with chronic heart failure (CHF) but little is known about its impact in AHF.

Methods Data were collected between April 2007 and March 2013 across 185 (> 95\%) hospitals in England and Wales from patients with a primary death or a discharge diagnosis of AHF. We investigated the association between the presence of AF and all-cause mortality during the index hospital admission, at 30 days and 1 year post-discharge.

Results Of 96,593 patients admitted with AHF, 44,642 (46\%) were in sinus rhythm (SR) and 51,951 (54\%) in AF. Patients with AF were older (mean age 79.8 (79.7-80) versus 74.7 (74.5-74.7) years; $p<0.001$ ), than those in SR. In a multivariable analysis, AF was independently associated with mortality at all time points, in hospital (HR 1.15, 95\% CI 1.09-1.21, $p<0.0001$ ), 30 days (HR 1.13, 95\% CI 1.08-1.19, $p<0.0001$ ), and 1 year (HR 1.09, 95\% CI 1.05-1.12, $p<0.0001$ ). In subgroup analyses, AF was independently associated with worse 30-day outcome irrespective of sex, ventricular phenotype and in all age groups except in those aged between 55 and 74 years.

Conclusion AF is independently associated with adverse prognosis in AHF during admission and up to 1 year post-discharge. As the clinical burden of concomitant AF and AHF increases, further refinement in the detection, treatment and prevention of AF-related complications may have a role in improving patient outcomes.
\end{abstract}

Keywords Acute heart failure $\cdot$ Atrial fibrillation $\cdot$ Prognosis $\cdot$ Mortality

\section{Introduction}

Atrial fibrillation (AF) is the commonest sustained arrhythmia in patients with heart failure (HF) with a prevalence reported between $30-50 \%$ in contemporary studies [1-4]. Previous studies have shown that the presence of AF in patients with $\mathrm{HF}$ is associated with an adverse prognosis,

Simon G. Anderson and Ahmad Shoaib contributed equally.

Simon G. Anderson and Ahmad Shoaib are co-first authors.

Electronic supplementary material The online version of this article (https://doi.org/10.1007/s00392-019-01463-5) contains supplementary material, which is available to authorized users.

Mamas A. Mamas

mamasmamas1@yahoo.co.uk

Extended author information available on the last page of the article although many of these studies have reported this association in patients with chronic stable HF [5]. A meta-analysis including 50,000 patients suggested that the presence of $\mathrm{AF}$ was associated with increased mortality risk in both randomised controlled studies and observational studies, irrespective of left ventricular (LV) function [6]. It appears that the risk is greatest in patients with incident $\mathrm{AF}$ compared to those with prevalent AF, RR: 2.21 versus 1.19 , respectively [7]. However, the prognostic impact of AF in patients admitted with an acute heart failure (AHF) is less clear [8-10].

Analysis of 10,701 patients hospitalised with an AHF as part of the EuroHeart Failure survey suggested that the presence of chronic AF did not impact in-hospital mortality (OR 0.84; 95\% CI 0.69-1.00) although new-onset AF was an independent predictor of in-hospital mortality (OR 1.53, 95\% CI 1.14-2.06) [11]. In contrast, in an analysis of 99,810 patients from 255 sites admitted with HF enrolled in Get With The Guidelines-Heart Failure (GWTG-HF) program 
in the United States, the presence of AF was an independent predictor of in-hospital mortality (OR 1.17, 95\% CI $1.05-1.29 ; p<0.005$ ) [7], whilst one other study has shown AF to be associated with worse outcomes in patients with AHF with underlying ischaemic heart disease (IHD) only [12]. Previous studies have not reported whether the prognostic impact of AF in patients admitted with an AHF is similar in patients with HFREF (Heart Failure with Reduced Ejection Fraction) and HFPEF (Heart Failure with Preserved Ejection Fraction), across genders or different age groups or have only reported in-hospital mortality outcomes with no post-discharge outcomes studied [7, 13-15].

We have, therefore, studied the association between AF and in-hospital and longer term mortality outcomes in an unselected cohort of patients admitted with an AHF in England and Wales through analysis of the National Heart Failure Audit. Furthermore, we aimed to examine whether the observed association differs in patients with HFPEF and HFREF.

\section{Methods}

The current study dataset is derived from around 150,000 patients hospitalised with heart failure and thus provides an excellent opportunity to study the clinical characteristics and outcomes in a 'real-world' setting. Mortality tracking is undertaken by the Medical Research Information Service using a patient's National Health Service (NHS) number, which provides a unique identifier for any person registered with the NHS in England and Wales, and the Office for National Statistics.

\section{Study population}

The National HF audit (NHFA) established in 2007 to monitor and improve care and treatment of patients is one of the largest HF cohorts in the world. 145 out of 150 NHS Trusts in England and Health Boards in Wales (97\%) submitted data to the audit between April 2012 and March 2013. The audit collects information from unscheduled individuals who have been admitted to participating hospitals with a primary death or discharge with a coded diagnosis of heart failure. This is designated by the following ICD codes: I11.0 hypertensive heart disease with (congestive) heart failure; I25.5 ischaemic cardiomyopathy; I42.0 dilated cardiomyopathy; I42.9 cardiomyopathy, unspecified; I50.0 congestive heart failure; I50.1 left ventricular failure or I50.9 heart failure, unspecified. The National Heart Failure Audit has developed a minimum data standard, in an attempt to ensure that the records submitted to the audit are fit for purpose.

For patients with more than one reported hospital admission, we randomly selected one admission. The analyses were restricted to patients with a diagnosis of atrial fibrillation or sinus rhythm on an ECG performed during the admission. Patients aged less than 18 years, duplicate records, no ECG data to clarify rhythm status and missing outcome data were excluded from the study (supplement Fig. 1). Evident moderate or severe left ventricular systolic dysfunction are derived from results of echocardiography, or other gold standard tests (including MRI, nuclear scan) during the current admission or in the 12 months prior to admission. Death was defined as mortality from any cause. Patients aged younger than 18, with missing records of ECG data or outcome data and those with no life status recorded were excluded.

\section{Primary outcome}

The primary outcomes were all-cause mortality as an inpatient, at 30 days, 1 year and all deaths.

\section{Covariates}

The National Health Service (NHS) information centre designed a secure, encrypted and web-based database called Lotus Notes for the recording of data relating to patients with heart failure which consisted of 233 fields. For the purpose of NHFA, 21 fields were assigned as core fields from this database and seven fields contained basic demographic information such as patient unique identifiers, gender and date of enrolment. The data were collected and recorded with the help of heart failure specialist nurses during each admission from patient case notes. Full details about selection of variables in this dataset and NHFA list of core fields are published elsewhere [16, 17].

\section{Statistical methods}

The analysis was performed using the Stata/MP 13.1 statistical software (College Station, TX). The cohort was stratified into two groups, those with sinus rhythm and those with atrial fibrillation. Where an individual had multiple admissions we randomly selected a single hospital episode. Continuous variables are presented as mean or $95 \%$ confidence intervals (CI), or median (interquartile range). Categorical variables are presented as counts (\%). We tested for differences between the groups using Chi-squared tests for nonparametric data and Student's $t$ test for normally distributed continuous variables.

We estimated hazard ratios (HRs) for mortality within 30-day using Cox regression with shared frailty or cluster models. A shared-frailty model is the survival-data analog to regression models with random effects and is used to model within-group correlation. Each hospital was assigned as a group variable within the model. 


\section{Covariates in multivariable models}

Multivariable adjustment included the continuous variables age as well as index of multiple deprivations and dichotomised categorical variables (yes versus no): sex (female versus male), history of myocardial infarction, hypertension, type 2 diabetes, ischaemic heart disease, valvular heart disease, LVH on echocardiography, and renal impairment. Other categorical variables included place of care, followup care, NYHA class and peripheral oedema. Separate subgroup analyses for LVSD, diastolic HF on echocardiography and gender as well as their interactions with the presence or absence of AF were performed.

To understand the association between age, sex, the interaction between age and sex, rhythm status and risk of allcause mortality at 1 year, we used the margins command in Stata to estimate margins of responses for specified values of covariates and present the results as a plot. Margins are statistics calculated from predictions of a previously fitted model (for example, a multivariable logistic regression) at fixed values of some covariates and averaging or otherwise integrating over the remaining covariates.

Sensitivity analyses were performed on models determined from the imputed data. Multiple imputations with chained equations were used to impute missing data. Five imputations were generated. Propensity score matching with nearest-neighbour matching was performed on the imputed cohort to create matched groups for presence or AF or sinus rhythm were used to estimate hazard ratios for mortality at 30-day, 1-year and full follow-up. We assessed the impact of heart rate as an effect modifier in a separate multivariable model. We employed the Stata module stddiff to estimate the standardised difference between groups It has been proposed that an absolute standardised difference of 0.10 or more indicates that covariates are imbalanced between groups [18].

Study findings are reported in accordance with the Strengthening the Reporting of Observational Studies in Epidemiology (STROBE) recommendations [19]. No ethics approval was needed for this analysis; the National Heart Failure Audit was conducted with the approval of the NHS Information Centre.

\section{Results}

A total of 168,843 patients were admitted with AHF in England and Wales between January 2007 and December 2013. Supplemental Fig. 1 illustrates a flowchart of eligibility and exclusion of patients included in the analysis. After exclusions, 96,593 records were available for analyses, of which 44,642 patients were in SR (46.5\%) and 51,951 (53.5\%) in AF.

Table 1 outlines the distribution of patient's characteristics by rhythm status. Patients with AF were significantly older than those with sinus rhythm [mean age 79.8 (79.7-80.0) vs 74.7 (74.5-74.7) years; $p<0.0001]$ and had a lower prevalence of diabetes, ischaemic heart disease and left ventricular systolic dysfunction (LVSD). The mean heart rate was greater in patients with AF 90 (89.5-90.3)] versus those with SR 84.0 (83.5-84.4); $p<0.0001$.

Table 2 illustrates differences in treatments, follow-up and mortality outcomes. Patients with AF were less likely to be prescribed an ACE inhibitor or ARB, but more likely
Fig. 1 Crude KM survival estimates

\section{Kaplan-Meier plot showing survival by heart rhythm status}

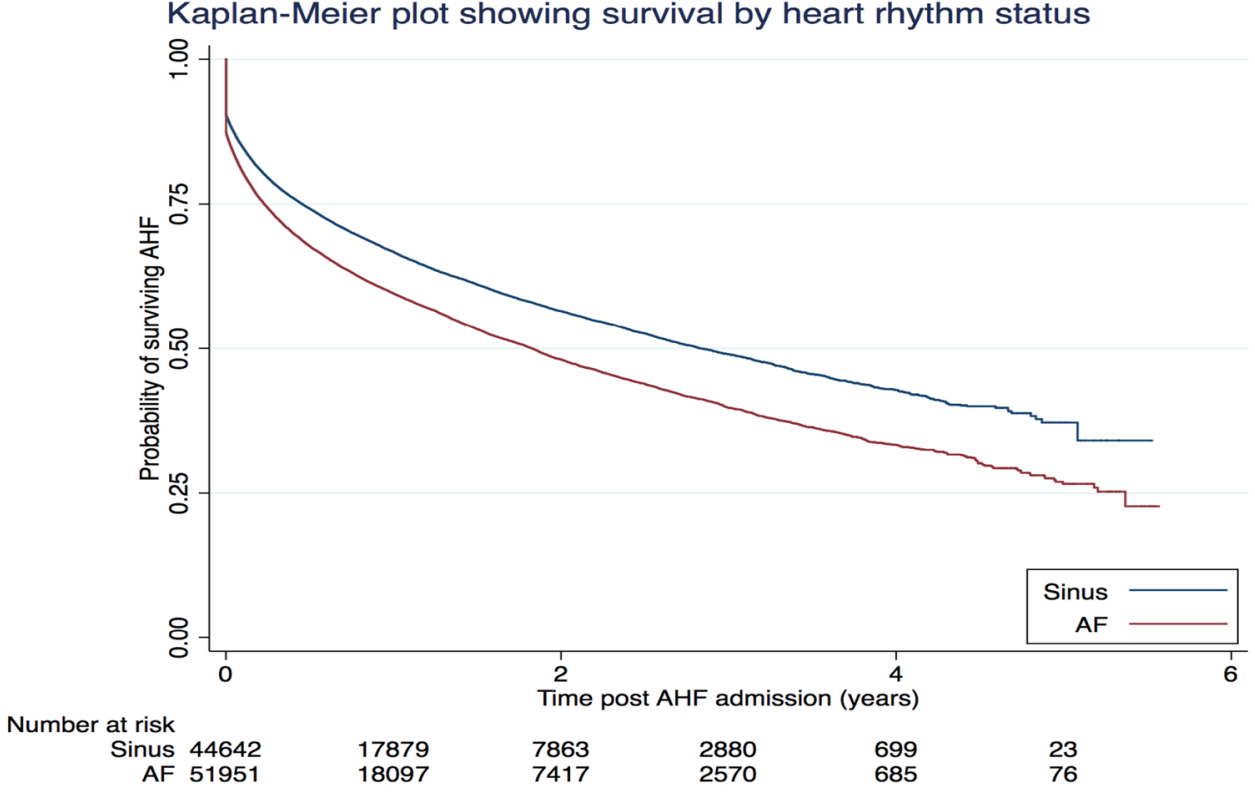


to be prescribed a B-blocker or a diuretic. No statistically significant difference was observed in median length of stay between the two cohorts [9 (IQR 4-17) versus 8 (IQR 4-15) days in patients with AF and SR, respectively].

Patients with SR were more likely to be managed in a cardiology ward and be followed up either by a heart failure service or a cardiologist (Table 2). In-hospital mortality was greater in patients with AF (11.5\%) compared to those patients with sinus rhythm $(8.6 \% ; p<0.0001)$ with similarly worse outcomes at 30 days $(17.4 \%$ vs $13.5 \%, p<0.0001)$ and 1 year $(36.6 \%$ vs $30.0 \%, p<0.0001)$. Figure 1 illustrates the crude Kaplan-Meier survival curves of both cohorts. Figure 2 in supplement shows variation in all-cause mortality by year of discharge from 2007 to 2013 and it remains highly consistent in AHF patients who have AF as compared to those who are in sinus rhythm.

Multivariable analyses were undertaken to assess relationships between admission rhythm, clinical outcomes and in-hospital, 30-day (Supplement Table 1) and 1-year mortality (Tables 3, 4, 5; Fig. 2). The presence of AF was independently associated with mortality at all time points studied; in-hospital (HR 1.15, 95\% CI 1.09-1.21, $p<0.0001$ ), 30-day (HR 1.13, 95\% CI 1.08-1.19, $p<0.0001$ ) and 1-year (HR 1.09, 95\% CI 1.05-1.12, $p<0.0001$ ) outcomes. Finally, Fig. 3 illustrates the relationship between atrial fibrillation and mortality across gender, the presence of LVSD, diastolic
Table 1 Distribution of patient characteristics by heart rhythm status

\begin{tabular}{|c|c|c|c|c|}
\hline Variable & & Atrial fibrillation & Sinus rhythm & \\
\hline & Total $^{\mathrm{b}}$ & $51,951(53.8)$ & $44,642(46.2)$ & \\
\hline Age (years) & 96,593 & $79.8(79.7-80.0)$ & $74.7(74.5-74.7)$ & $<0.0001$ \\
\hline \multicolumn{5}{|l|}{ Age categories (years) } \\
\hline Min-54 & $5749 / 96,593$ & $1521(2.9)$ & $4228(9.5)$ & $<0.0001$ \\
\hline $55-64$ & $8552 / 96,593$ & $3299(6.3)$ & $5253(11.8)$ & $<0.0001$ \\
\hline $65-74$ & $18,524 / 96,593$ & $8845(17.0)$ & 9679 (21.7) & $<0.0001$ \\
\hline $75-84$ & $34,238 / 96,593$ & $19,623(37.8)$ & $14,615(32.7)$ & $<0.0001$ \\
\hline $85+$ & $29,530 / 96,593$ & $18,663(36.0)$ & $10,867(24.3)$ & $<0.0001$ \\
\hline \multicolumn{5}{|l|}{ Sex } \\
\hline Male & $52,271 / 96,552$ & $28,059(54.0)$ & $24,212(54.3)$ & 0.4925 \\
\hline Female & $44,281 / 96,552$ & $23,870(46.0)$ & $20,411(45.7)$ & 0.5277 \\
\hline Previous AMI & $27,276 / 89,601$ & $12,538(26.3)$ & $14,738(35.1)$ & $<0.0001$ \\
\hline History of diabetes & $27,267 / 92,550$ & $13,315(26.9)$ & $13,952(32.4)$ & $<0.0001$ \\
\hline History of hypertension & $49,972 / 90,884$ & $27,189(55.9)$ & $22,783(53.9)$ & $<0.0001$ \\
\hline History of IHD & $42,387 / 90,874$ & $21,690(44.8)$ & $20,697(48.7)$ & $<0.0001$ \\
\hline History of valvular heart disease & $19,641 / 88,285$ & $12,158(25.8)$ & $7483(18.2)$ & $<0.0001$ \\
\hline \multicolumn{5}{|l|}{ NYHA class } \\
\hline Class I & $5,294 / 88,888$ & $2177(4.6)$ & $3117(7.5)$ & $<0.0001$ \\
\hline Class II & $14,850 / 88,888$ & $7348(15.4)$ & $7502(18.2)$ & $<0.0001$ \\
\hline Class III & $39,210 / 88,888$ & $21,316(44.8)$ & $17,894(43.3)$ & $<0.0001$ \\
\hline Class IV & $29,534 / 88,888$ & $16,717(35.2)$ & $12,817(31.0)$ & $<0.0001$ \\
\hline \multicolumn{5}{|l|}{ Peripheral oedema } \\
\hline None & $21,022 / 86,660$ & 8965 (19.4) & $12,057(29.9)$ & $<0.0001$ \\
\hline Mild & $22,267 / 86,660$ & $11,752(25.4)$ & $10,515(26.0)$ & $<0.0001$ \\
\hline Moderate & $28,041 / 86,660$ & $16,061(34.7)$ & $11,980(29.7)$ & $<0.0001$ \\
\hline Severe & $15,330 / 86,660$ & $9,513(20.5)$ & $5,817(14.4)$ & $<0.0001$ \\
\hline Moderate or severe LVSD ${ }^{\mathrm{a}}$ & $54,475 / 79,724$ & $27,577(65.2)$ & $26,898(71.9)$ & $<0.0001$ \\
\hline Heart rate (bpm) & 20,976 & $90.0(89.5-90.3)$ & $84.0(83.5-84.4)$ & $<0.0001$ \\
\hline
\end{tabular}

Column percentages are presented

$p$ values from Student's $t$ tests, two-sample test of proportions or Chi-squared tests for differences in proportions for atrial fibrillation versus sinus rhythm respectively

$A M I$ acute myocardial infarction, IHD ischaemic heart disease, NYHA New York Heart Association, LVSD left ventricle systolic dysfunction

${ }^{a}$ Only 20,976 participants had heart rate data recorded of which 9400 were in sinus rhythm and 11,576 were in $\mathrm{AF}$

${ }^{\mathrm{b}}$ Due to missing data, the percentages reported are from these totals 
dysfunction and age. AF was independently associated with worse 30-day mortality outcomes in all subgroups studied, except patients aged 55-64 and 65-74, where statistical trends that were not significant were observed (HR 1.04 95\% CI 0.85-1.29, $p=0.69$ and HR $1.0995 \%$ CI 0.97-1.22).

The impact of heart rate as an effect modifier is assessed in a separate multivariable model. Only around a quarter of the study population had heart rate data recorded. We assessed the standardised difference between groups (of individuals who had heart rate recorded $(n=20,976)$ and those who did not have heart recorded, $n=75,617$ ) for both continuous and categorical variables. A table of these standardised differences are presented in Supplement Table 2. The impact of increased HR was assessed in a series of models in the 20,976 patients who had the data. Increased heart rate (HR increase by 10 beats per min) is associated with high inpatient deaths but lost its significance for long-term mortality (Supplement Tables 3, 4). AF is independently associated with adverse prognosis whether HR data are recorded or not in separate multivariable Cox regression models on imputed data. (Supplement Tables 5, 6). However, in a multivariable model from propensity score-matched (PSM) data in those with HR data, the adverse significant effect of AF was lost, likely due to type 2 errors from the small numbers present in model (Supplement table 7).

We assessed the interaction of gender or age with $\mathrm{AF}$ and its impact on 1-year mortality in a separate multivariable logistic regression analysis and results are presented in
Table 2 Treatment, follow-up and mortality status by heart rhythm status

\begin{tabular}{|c|c|c|c|c|}
\hline Variable & & Atrial fibrillation & Sinus rhythm & $p$ value \\
\hline & Total & & & \\
\hline \multicolumn{5}{|l|}{ Treatment medications } \\
\hline MRA & $29,402 / 80,939(36.3)$ & $15,776(36.7)$ & $13,626(35.9)$ & 0.017 \\
\hline $\mathrm{ARB}$ & $11,651 / 74,027(15.7)$ & $6091(15.5)$ & $5560(16.0)$ & 0.058 \\
\hline ACE inhibitor & $50,168 / 79,171(63.4)$ & $25,821(61.6)$ & $24,347(65.4)$ & $<0.0001$ \\
\hline ACE inhibitor OR ARB & $61,165 / 80,903(75.6)$ & $31,598(73.8)$ & 29,567 (77.6) & $<0.0001$ \\
\hline Beta-blocker & $53,262 / 80,864(65.9)$ & $28,546(66.3)$ & $24,716(65.3)$ & 0.003 \\
\hline Thiazide diuretic & $3647 / 79,869(4.6)$ & $2136(5.0)$ & $1511(4.1)$ & $<0.0001$ \\
\hline Digoxin & $20,719 / 81,510(25.4)$ & $18,107(40.9)$ & $2612(7.0)$ & $<0.0001$ \\
\hline Loop diuretic & $77,373 / 87,151(88.8)$ & $42,291(90.9)$ & $35,082(86.4)$ & $<0.0001$ \\
\hline Median length of stay in days (IQR) & $8(4-16)$ & $9(4-17)$ & $8(4-15)$ & $<0.0001$ \\
\hline \multicolumn{5}{|l|}{ Discharge medications } \\
\hline Beta-blocker & $36,494 / 58,282(62.6)$ & $19,193(62.7)$ & $17,301(62.6)$ & $<0.0001$ \\
\hline Digoxin & $15,134 / 58,514(25.8)$ & $13,185(42.2)$ & $1949(7.2)$ & $<0.0001$ \\
\hline Loop diuretic & $55,140 / 62,531(88.2)$ & $29,820(90.3)$ & $25,320(85.8)$ & $<0.0001$ \\
\hline Thiazide & $2558 / 58,385(4.4)$ & $1493(4.8)$ & $1065(3.8)$ & $<0.0001$ \\
\hline $\mathrm{ARB}$ & $8305 / 54,425(15.3)$ & $4307(15.0)$ & $3998(15.5)$ & $<0.0001$ \\
\hline MRA & $20,285 / 58,548(34.6)$ & $10,776(35.1)$ & $9509(34.2)$ & 0.025 \\
\hline ACE inhibitor & $36,326 / 57,635(63.0)$ & $18,468(61.2)$ & $17,858(65.1)$ & $<0.0001$ \\
\hline ACE inhibitor OR ARB & $44,125 / 58,709(75.2)$ & $22,541(73.3)$ & $21,584(77.2)$ & $<0.0001$ \\
\hline \multicolumn{5}{|l|}{ Main place of care } \\
\hline Cardiology & $43,290 / 95,963(45.1)$ & $21,952(42.5)$ & $21,338(48.1)$ & $<0.0001$ \\
\hline General medicine & $42,203 / 95,963(44.0)$ & $23,777(46.1)$ & $18,426(41.6)$ & $<0.0001$ \\
\hline Other & $10,470 / 95,963(10.9)$ & $5905(11.4)$ & $4565(10.3)$ & 0.0735 \\
\hline \multicolumn{5}{|l|}{ Follow-up } \\
\hline Heart failure liaison service & $43,807 / 88,852(47.3)$ & $22,303(46.9)$ & $21,504(52.0)$ & $<0.0001$ \\
\hline Palliative care & $3,822 / 87,934(4.4)$ & $2242(4.8)$ & $1579(3.8)$ & $<0.0001$ \\
\hline Care of the elderly & $12,279 / 88,951(13.8)$ & $6859(14.4)$ & $5420(13.1)$ & $<0.0001$ \\
\hline Cardiology & $42,497 / 89,885(49.3)$ & $21,127(44.0)$ & $21,370(51.0)$ & $<0.0001$ \\
\hline GP follow-up & $62,072 / 89,298(69.5)$ & 33,107 (69.2) & 28,965 (69.8) & 0.055 \\
\hline
\end{tabular}

Column percentages are presented

$p$ values from Student's $t$ tests, two-sample test of proportions or Chi-squared tests for differences in proportions for atrial fibrillation versus sinus rhythm respectively

$M R A$ mineralocorticoid receptor antagonist, $A R B$ angiotensin receptor blockers, $A C I$ angiotensin-converting enzyme inhibitor, $I Q R$ interquartile range 
the form of margin plots (Supplement Fig. 3). Essentially, adverse effects of age and male gender on HF patients who have AF are minimal up to 60 years of age compared to those in sinus rhythm but increases clearly after it.

\section{Discussion}

In this large multicentre national heart failure registry, we demonstrate that the presence of AF in patients admitted with an AHF is independently associated with an increased risk of in-hospital, 30-day and 1-year mortality. This relationship is observed in both HFREF and HFPEF, across genders and across different age groups in multivariate analyses.

These are the first data assessing the prognosis of AF in AHF during index hospital admission, 30 days and 1 year after discharge with detailed analysis in clinically important subgroups. The NHFA is one of the largest HF registries in the world providing more pragmatic real-world data as compared to post hoc analysis of clinical trials which are conducted in controlled clinical settings. No prior studies have evaluated the effect of AF in AHF patients across both genders and different age groups in the short and longer terms. Our study demonstrates significant differences in all clinical outcomes between patients with sinus rhythm and AF. Patients with HF who were found to be in AF have fewer comorbidities on presentation, prolonged hospital stay and worse short- and long-term mortalities in multivariable analysis. The results of this study are in contrast to previous smaller studies where AF was not associated with adverse long-term outcomes. For instance, in the Danish Investigations of Arrhythmia and Mortality on Dofetilide (DIAMOND) study (3587 patients), the authors reported similar in-hospital mortality between patients presenting in AF and sinus rhythm [12]. An analysis of the national GWTG-HF registry data $(99,810$ patients from 255 sites of US) also demonstrated a clear association between AF and mortality in hospitalised heart failure patients but no post-discharge follow-up data were available [7]. Our study represents a more contemporary cohort of patients (years of data collection 2007-2013) as compared to previously published research work (EHFS1 2000-2001, GWTG-HF 2005-2010, ASCEND-HF 2007-2010) [7, 11, 20]. Furthermore, our study also provides important information about processes of care in the treatment of these high-risk patients for the first time, such as their main place of care during hospital and post-discharge follow-up. It is pertinent to note that majority of these high-risk group patients with $\mathrm{AF}$ are neither treated in a cardiology ward (44\%) nor followed up in cardiology clinics after discharge (47\%). This finding is important and may provide a mechanism that contributes to our observations that patients with AF have adverse outcomes particularly in the longer term as previous work has demonstrated improved survival if HF patients are treated in cardiology wards or are followed up in either heart failure or cardiology clinics [21].

The relationship between AF and HF was first described in the literature almost 100 years ago [22]. This association could be explained to some extent by the presence of common risk factors such as age, diabetes, hypertension,
Fig. 2 Hazard ratios for mortality from multivariate analysis for atrial fibrillation versus sinus rhythm at different time points (imputed data)

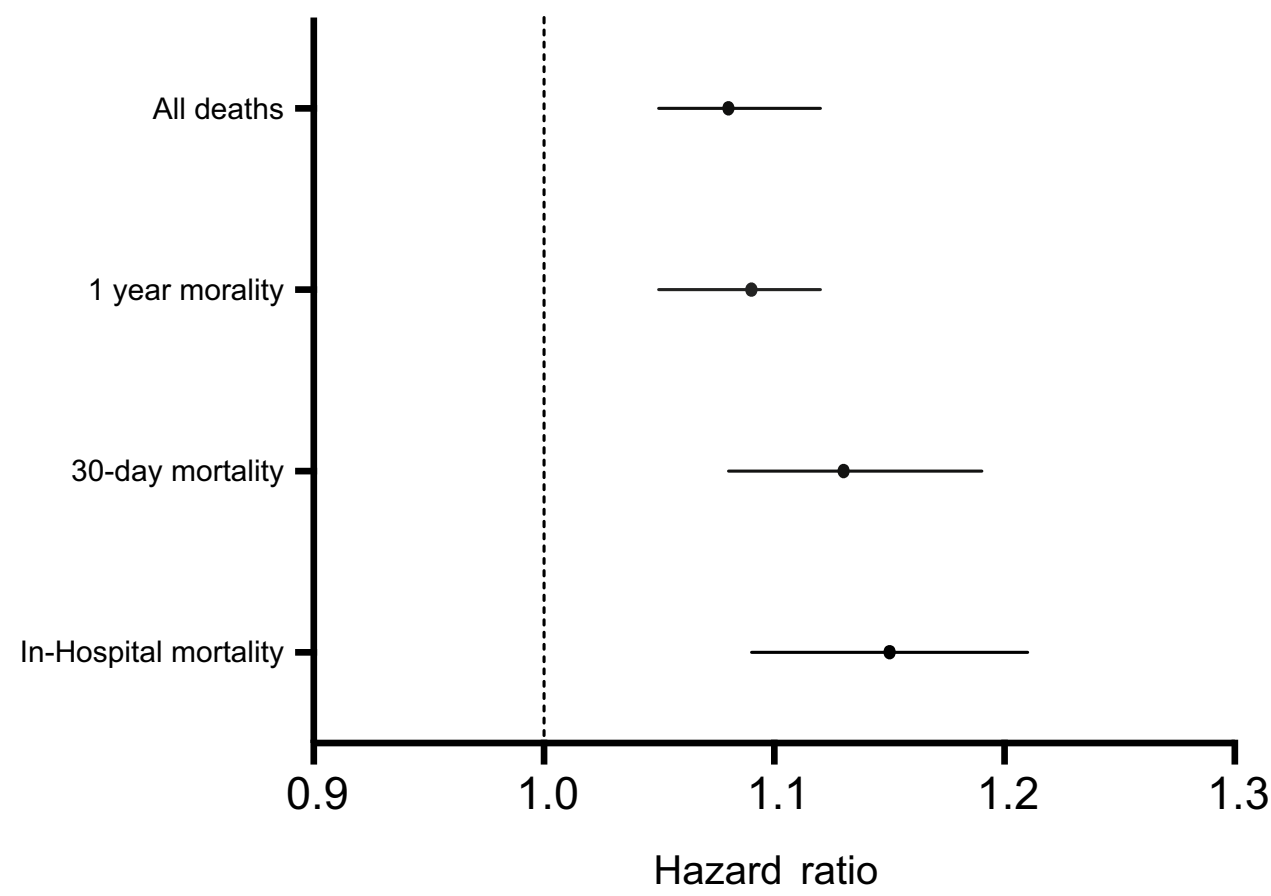


Table 3 Multivariate analyses for relation between AF (versus sinus rhythm) and 1-year mortality on imputed data of patients recorded as $\mathrm{HF},(N=96,593)$

\begin{tabular}{|c|c|c|c|c|}
\hline \multirow[b]{2}{*}{ Atrial fibrillation on ECG } & \multirow{2}{*}{$\frac{\mathrm{HR}}{1.09}$} & \multicolumn{2}{|c|}{$95 \% \mathrm{CI}$} & \multirow{2}{*}{$\frac{p \text { value }}{<0.001}$} \\
\hline & & 1.05 & 1.12 & \\
\hline \multicolumn{5}{|l|}{ Breathlessness } \\
\hline NYHA I & 1.00 & & & \\
\hline NYHA II & 1.05 & 0.96 & 1.16 & 0.292 \\
\hline NYHA III & 1.16 & 1.06 & 1.27 & 0.002 \\
\hline NYHA IV & 1.30 & 1.19 & 1.42 & 0 \\
\hline \multicolumn{5}{|l|}{ Peripheral oedema } \\
\hline None & 1.00 & & & \\
\hline Mild & 1.08 & 1.02 & 1.14 & 0.01 \\
\hline Moderate & 1.23 & 1.17 & 1.30 & $<0.0001$ \\
\hline Severe & 1.47 & 1.40 & 1.55 & $<0.0001$ \\
\hline Palliative care follow-up & 2.54 & 2.35 & 2.74 & $<0.0001$ \\
\hline Heart failure liaison service & 0.84 & 0.79 & 0.88 & $<0.0001$ \\
\hline GP follow-up & 0.60 & 0.56 & 0.64 & $<0.0001$ \\
\hline Care of the elderly follow-up & 0.80 & 0.76 & 0.85 & $<0.0001$ \\
\hline Cardiology follow-up & 0.60 & 0.57 & 0.63 & $<0.0001$ \\
\hline Previous AMI & 1.14 & 1.09 & 1.18 & $<0.0001$ \\
\hline History of diabetes & 1.05 & 1.02 & 1.08 & 0.003 \\
\hline History of hypertension & 0.93 & 0.90 & 0.95 & $<0.0001$ \\
\hline History of IHD & 1.12 & 1.08 & 1.16 & $<0.0001$ \\
\hline History of valvular heart disease & 1.24 & 1.19 & 1.29 & $<0.0001$ \\
\hline \multicolumn{5}{|l|}{ Age categories (years) } \\
\hline Min-54 & 1.00 & & & \\
\hline $55-64$ & 1.43 & 1.27 & 1.59 & $<0.0001$ \\
\hline $65-74$ & 2.02 & 1.83 & 2.24 & $<0.0001$ \\
\hline $75-84$ & 2.74 & 2.47 & 3.04 & $<0.0001$ \\
\hline $85+$ & 3.68 & 3.30 & 4.10 & $<0.0001$ \\
\hline Male & 1.14 & 1.11 & 1.17 & $<0.0001$ \\
\hline LVH & 0.86 & 0.81 & 0.92 & $<0.0001$ \\
\hline ACEi/ARB use & 0.66 & 0.64 & 0.69 & $<0.0001$ \\
\hline Beta-blocker & 0.78 & 0.75 & 0.82 & $<0.0001$ \\
\hline Thiazide & 1.12 & 1.05 & 1.20 & 0.001 \\
\hline Loop diuretic & 0.72 & 0.67 & 0.77 & $<0.0001$ \\
\hline Digoxin & 0.89 & 0.85 & 0.92 & $<0.0001$ \\
\hline Renal failure & 1.14 & 1.08 & 1.22 & $<0.0001$ \\
\hline Length of time in hospital (per 5 days) & 1.02 & 1.02 & 1.03 & $<0.0001$ \\
\hline
\end{tabular}

$H R$ hazard ratio, $C I$ confidence interval, ECG electrocardiograph, NYHA New York Heart Association, AMI acute myocardial infarction, $G P$ general practitioner, $I H D$ ischaemic heart disease, $L V H$ left ventricular hypertrophy, $A R B$ angiotensin receptor blockers, $A C I$ angiotensin-converting enzyme inhibitor

high BMI, valvular, ischaemic and structural heart disease [23]. These risk factors contribute to myocardial cellular and extracellular damage, neurohormonal and electrophysiological changes which predispose the heart to AF and heart failure $[24,25]$. The increased resting and exaggerated exercise heart rate response in $\mathrm{AF}$ predisposes to shorter diastolic
Table 4 Multivariate analyses for relation between AF (versus sinus rhythm) and in-hospital mortality on imputed data of patients recorded as $\mathrm{HF}(N=96,593)$

\begin{tabular}{lcccc}
\hline & HR & $95 \%$ & CI & $p$ value \\
\hline Atrial fibrillation on ECG & 1.15 & 1.09 & 1.21 & $<0.0001$ \\
Breathlessness & & & & \\
NYHA I & 1.00 & & & \\
NYHA II & 1.01 & 0.87 & 1.18 & 0.896 \\
NYHA III & 1.10 & 0.96 & 1.27 & 0.18 \\
NYHA IV & 1.32 & 1.14 & 1.52 & $<0.0001$ \\
Peripheral oedema & & & & \\
None & 1.00 & & & \\
Mild & 1.03 & 0.94 & 1.13 & 0.498 \\
Moderate & 1.09 & 1.01 & 1.18 & 0.023 \\
Severe & 1.33 & 1.22 & 1.45 & $<0.0001$ \\
Palliative care follow-up & 1.64 & 1.43 & 1.89 & $<0.0001$ \\
Heart failure liaison service & 0.51 & 0.44 & 0.59 & $<0.0001$ \\
GP follow-up & 0.23 & 0.19 & 0.27 & $<0.0001$ \\
Care of the elderly follow-up & 0.44 & 0.37 & 0.52 & $<0.0001$ \\
Cardiology follow-up & 0.33 & 0.28 & 0.39 & $<0.0001$ \\
Previous AMI & 1.05 & 0.99 & 1.11 & 0.123 \\
History of diabetes & 1.01 & 0.96 & 1.06 & 0.799 \\
History of hypertension & 0.97 & 0.92 & 1.01 & 0.156 \\
History of IHD & 1.08 & 1.03 & 1.13 & 0.003 \\
History of valvular heart disease & 1.16 & 1.10 & 1.23 & $<0.0001$ \\
Age categories (years) & & & & \\
Min-54 & 1.00 & & & \\
55-64 & 1.38 & 1.13 & 1.70 & 0.002 \\
65-74 & 1.88 & 1.55 & 2.28 & $<0.0001$ \\
75-84 & 2.33 & 1.90 & 2.84 & $<0.0001$ \\
85+ & 2.72 & 2.21 & 3.36 & $<0.0001$ \\
Male & 1.06 & 1.02 & 1.11 & 0.003 \\
LVH & 0.88 & 0.80 & 0.97 & 0.01 \\
ACEi/ARB use & 0.77 & 0.72 & 0.83 & $<0.0001$ \\
Beta-blocker & 0.80 & 0.75 & 0.86 & $<0.0001$ \\
Thiazide & 0.91 & 0.78 & 1.06 & 0.205 \\
Loop diuretic & 0.70 & 0.65 & 0.76 & $<0.0001$ \\
Digoxin & 0.70 & 0.63 & 0.79 & $<0.0001$ \\
Renal failure & 1.06 & 1.25 & 0.003 \\
Length of time in hospital (per 5 days) & 1.02 & $<0.0001$ \\
\hline
\end{tabular}

$H R$ hazard ratio, $C I$ confidence interval, ECG electrocardiograph, NYHA New York Heart Association, AMI acute myocardial infarction, GP general practitioner, $I H D$ ischaemic heart disease, $L V H$ left ventricular hypertrophy, $A R B$ angiotensin receptor blockers, $A C I$ angiotensin-converting enzyme inhibitor

filling time which leads to a reduction in cardiac output [26]. Furthermore, loss of effective atrial contraction also contributes to reduce diastolic filling. AF is considered the most common cause of tachycardia-induced cardiomyopathy. In a similar way, HF can increase the risk for the development of AF by raising cardiac filling pressures, autonomic 
Table 5 Multivariate analyses for relation between AF (versus sinus rhythm) and all deaths on imputed data of patients recorded as HF, $(N=96,593)$

\begin{tabular}{|c|c|c|c|c|}
\hline \multirow[b]{2}{*}{ Atrial fibrillation on ECG } & \multirow{2}{*}{$\frac{\mathrm{HR}}{1.08}$} & \multicolumn{2}{|c|}{$95 \% \mathrm{CI}$} & \multirow{2}{*}{$\frac{p \text { value }}{<0.0001}$} \\
\hline & & 1.05 & 1.12 & \\
\hline \multicolumn{5}{|l|}{ Breathlessness } \\
\hline NYHA I & 1.00 & & & \\
\hline NYHA II & 1.07 & 0.98 & 1.16 & 0.123 \\
\hline NYHA III & 1.17 & 1.07 & 1.27 & $<0.0001$ \\
\hline NYHA IV & 1.30 & 1.19 & 1.41 & $<0.0001$ \\
\hline \multicolumn{5}{|l|}{ Peripheral oedema } \\
\hline None & 1.00 & & & \\
\hline Mild & 1.08 & 1.03 & 1.14 & 0.002 \\
\hline Moderate & 1.23 & 1.18 & 1.29 & $<0.0001$ \\
\hline Severe & 1.46 & 1.39 & 1.53 & $<0.0001$ \\
\hline Palliative care follow-up & 2.48 & 2.28 & 2.69 & $<0.0001$ \\
\hline Heart failure liaison service & 0.87 & 0.83 & 0.91 & $<0.0001$ \\
\hline GP follow-up & 0.65 & 0.61 & 0.69 & $<0.0001$ \\
\hline Care of the elderly follow-up & 0.83 & 0.78 & 0.87 & $<0.0001$ \\
\hline Cardiology follow-up & 0.63 & 0.60 & 0.66 & $<0.0001$ \\
\hline Previous AMI & 1.13 & 1.09 & 1.17 & $<0.0001$ \\
\hline History of diabetes & 1.07 & 1.04 & 1.10 & $<0.0001$ \\
\hline History of hypertension & 0.92 & 0.90 & 0.95 & $<0.0001$ \\
\hline History of IHD & 1.13 & 1.09 & 1.17 & $<0.0001$ \\
\hline History of valvular heart disease & 1.22 & 1.18 & 1.26 & $<0.0001$ \\
\hline \multicolumn{5}{|l|}{ Age categories (years) } \\
\hline Min-54 & 1.00 & & & \\
\hline $55-64$ & 1.47 & 1.33 & 1.62 & $<0.0001$ \\
\hline $65-74$ & 2.11 & 1.93 & 2.31 & $<0.0001$ \\
\hline $75-84$ & 2.89 & 2.63 & 3.18 & $<0.0001$ \\
\hline $85+$ & 4.00 & 3.63 & 4.41 & $<0.0001$ \\
\hline Male & 1.14 & 1.11 & 1.17 & $<0.0001$ \\
\hline LVH & 0.89 & 0.83 & 0.94 & $<0.0001$ \\
\hline ACEi/ARB use & 0.68 & 0.65 & 0.70 & $<0.0001$ \\
\hline Beta-blocker & 0.78 & 0.75 & 0.81 & $<0.0001$ \\
\hline Thiazide & 1.13 & 1.06 & 1.20 & $<0.0001$ \\
\hline Loop diuretic & 0.76 & 0.71 & 0.81 & $<0.0001$ \\
\hline Digoxin & 0.90 & 0.87 & 0.93 & $<0.0001$ \\
\hline Renal failure & 1.13 & 1.06 & 1.20 & 0.002 \\
\hline Length of time in hospital (per 5 days) & 1.02 & 1.02 & 1.03 & $<0.0001$ \\
\hline
\end{tabular}

$H R$ hazard ratio, $C I$ confidence interval, $E C G$ electrocardiograph, NYHA New York Heart Association, AMI acute myocardial infarction, $G P$ general practitioner, $I H D$ ischaemic heart disease, $L V H$ left ventricular hypertrophy, $A R B$ angiotensin receptor blockers, $A C I$ angiotensin-converting enzyme inhibitor

and neuroendocrine dysregulation and increased interstitial fibrosis [27]. Despite the well-established association between these two conditions, less is known about the effects of AF particularly in HFPEF and HFREF on acute and longterm prognosis in patients hospitalised with heart failure.
Data that examine the relationship between $\mathrm{AF}$ and clinical outcomes in HF patients have many limitations and report inconsistent results. For instance, a retrospective analysis of SOLVD (Studies Of Left Ventricle Dysfunction) trial suggested that baseline AF was an independent predictor for all-cause mortality and the combined endpoint of death and readmission due to HF in patients who had ejection fraction $<35 \%$ [28]. Similar results were reported in the VALIANT trial (Valsartan in acute myocardial infarction) where AF was also associated with long-term mortality in acute myocardial infarction patients complicated by HF [28]. In contrast, a retrospective analysis of the Carvedilol or Metoprolol European Trial (COMET) data demonstrated that baseline AF significantly increased the risk of death and HF hospitalization in patients who had ejection fraction $<35 \%$, although this association lost its significance in multivariate analysis [29].

Data around the prognostic impact of AF in patients with AHF are more limited. A retrospective analysis of Acute Study of Clinical Effectiveness of Nesiritide in Decompensated Heart Failure (ASCEND-HF) trial showed that the patients admitted to hospital with AHF, current or background history of AF are independently associated with lesser dyspnoea improvement, high mortality and morbidity (adjusted odds ratio 1.19, CI 1.02-1.38, $p=0.029)$ as compared to those who are in sinus rhythm [20]. Furthermore, in GWTG-HF study, the presence of AF was an independent predictor of in -hospital mortality and more prolonged index hospital admission ( $>4$ days) compared to sinus rhythm. However, in contrast to our analysis, no post-discharge mortality data were available. Moreover, the present analysis is more robust as compared to all other research on this subject as this study demonstrated that adverse effects of AF remain significant across gender and different age groups.

The relationship between AF and clinical outcomes in patients with HF may relate to the severity of LV dysfunction. For instance, Middlekauf et al. reported that AF was a stronger predictor of adverse outcomes (total mortality and sudden death) in patients with mild to moderate heart failure as compared to those with severe heart failure [30]. Similarly, Linssen et al. found that AF was associated with higher NT-proBNP levels, mortality and morbidity in HFPEF but not in HFREF patients [31]. In contrast, in a systematic review and meta-analysis of over 54,000 patients, Kotecha et al. reported that all-cause mortality was significantly higher in patients with AF and HFREF compared to HFPEF [32]. Our analysis has suggested that the adverse effect of AF remain significant in both HFPEF and HFREF in a cohort of 96,000 patients during hospital admission and 30 days after discharge.

The relationship between AF and outcomes is further complicated by the chronicity of AF. For example, 
Fig. 3 Sub-group analyses for risk of mortality at 30 days in $\mathrm{AF}$ versus sinus rhythm. $H F$ heart failure, $L V S D$ left ventricle systolic dysfunction

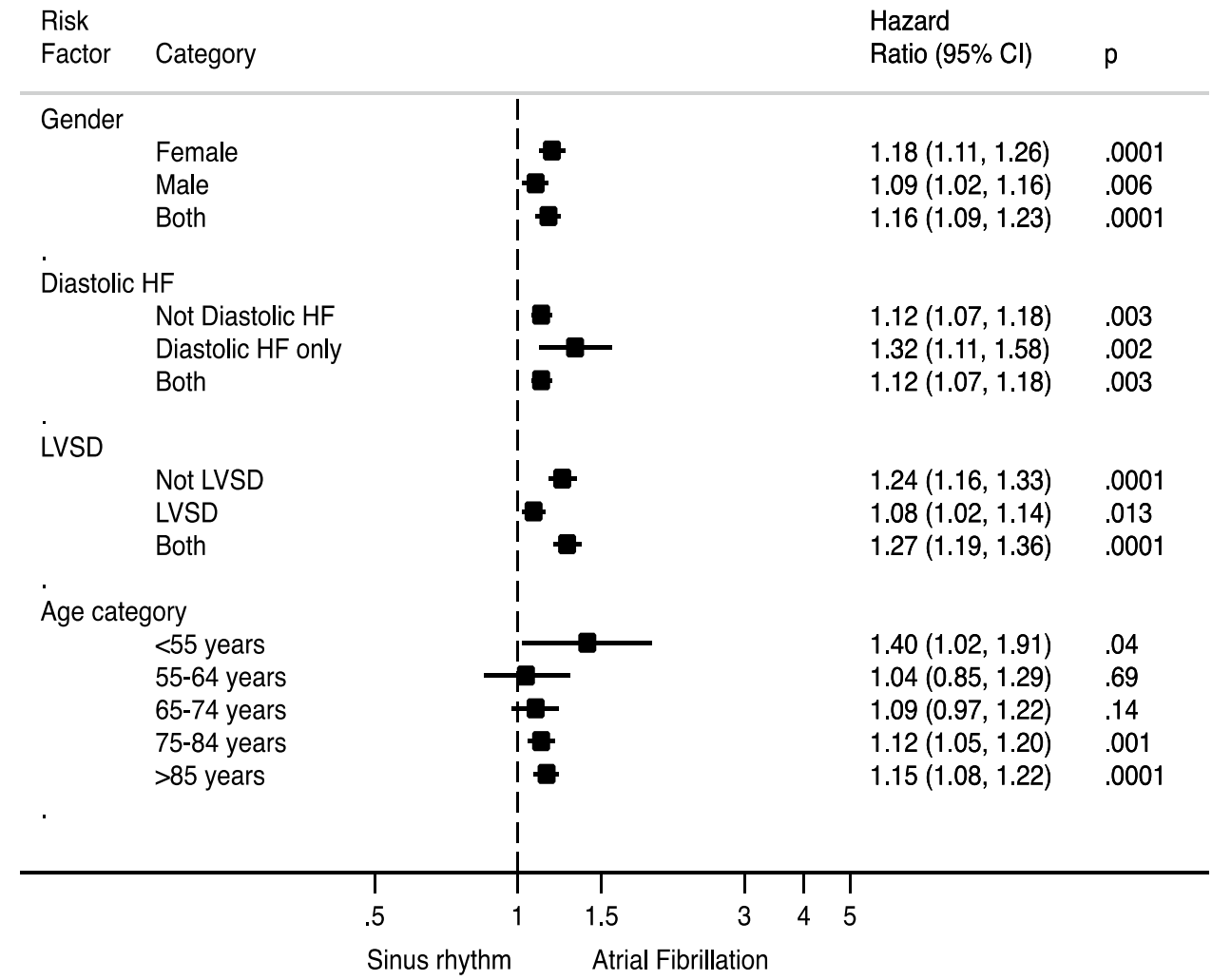

new-onset and not chronic AF was an independent predictor of all-cause mortality in the EHFS1 and COMET studies [33]. As outlined, previous work has suggested that incident AF has a greater prognostic impact than chronic AF and established therapies for HF may reduce the incident AF risk. Retrospective analysis of large RCTs suggests that ACEI and ARB (angiotensin-converting enzyme inhibitors and angiotensin receptor blocker) can reduce the risk of incident AF in HF patients $[34,35]$. However, this preventive role of ACEI/ARB is less evident in $\operatorname{HFPEF}[36,37]$. Initiation of beta-blocker (BB) therapy in HFREF patients who were pre-treated with ACEI/ARB was associated with one-third reduction of new-onset AF, [38] although BB does not reduce either mortality or hospital admission in patients who have HFREF and AF [38, 39]. Eplerenone also reduced the risk of incident AF in HF patients with $\mathrm{LVEF}<35 \%$ when added to ACEI/ARB and BB [40]. In our study, a similar proportion of patients received $\mathrm{BB}$ at discharge in both groups $(63 \%)$ but the AF cohort received less ACEI/ARB (76\% versus $81 \%$ ).

\section{Limitations}

As with any observational study, there are a number of limitations. Whilst we have attempted to adjust for differences in clinical characteristics and patient demographics in the current work, the National Heart Failure audit does not collect data on co-morbid burden and frailty that is known to influence clinical outcomes in patients with AHF. Missing data is a common problem in large dataset studies which varied in extent depending on the study variable. However, we tried to approximate these values using multiple imputations to impute the missing variables. Prior history of AF was not recorded in NHFA dataset. Therefore, we are unable to differentiate between new-onset and chronic AF, as some previous studies report that the adverse prognostic impact of AF is limited to new-onset AF [7, 11]. Furthermore, data regarding the aetiology of heart failure are not recorded in this registry, and so it is unknown whether the prognostic impact of AF differs between the ischaemic and non-ischemic aetiologies of HF. Heart Failure with Midrange ejection fraction (HFmrEF) is a recently defined entity in the European Society of Cardiology (ESC) 2016 HF guidelines, which encompass those patients who have a LVEF of 40-49\%, elevated levels of natriuretic peptides and either LV hypertrophy, left atrium enlargement or diastolic dysfunction [41]. We were unable to analyse our dataset for this category as our data were collected before this publication [42]. 


\section{Conclusion}

The current report, of approximately 100,000 unselected emergency hospital admissions due to heart failure, is the first to compare survival outcomes in AHF patients in the presence or absence of AF during an index hospital admission, at 30 days and 1 year after discharge, across gender and in different age groups. This study reveals that AF is associated with high mortality in AHF during hospital admission and up to 1 year after discharge after taking into account many potential confounders. Patients with AF in AHF are a high-risk cohort, although it is unclear whether targeting AF may improve outcomes in this cohort of patients, or whether it represents a marker of disease severity.

Author contributions SGA, PKM, and MAM: conception and design or analysis and interpretation of data. SGA, AS, PKM, JGC, SMH, TAM, HD, BK, CJG, and MAM: drafting of the manuscript and revising it critically for important intellectual content. AS, PKM, JGC, $\mathrm{SMH}$, TAM, HD, BK, CJG, and MAM: final approval of the manuscript submitted.

\section{Compliance with ethical standards}

Conflict of interest The authors confirm that there are no relationships with industry or financial associations that might pose a conflict of interest in connection with this article.

Open Access This article is distributed under the terms of the Creative Commons Attribution 4.0 International License (http://creativeco mmons.org/licenses/by/4.0/), which permits unrestricted use, distribution, and reproduction in any medium, provided you give appropriate credit to the original author(s) and the source, provide a link to the Creative Commons license, and indicate if changes were made.

\section{References}

1. McMurray JJV, Packer M, Desai AS, Gong J, Lefkowitz MP, Rizkala AR et al (2014) Angiotensin-neprilysin inhibition versus enalapril in heart failure. N Engl J Med 371(11):993-1004

2. Zannad F, McMurray JJV, Krum H, van Veldhuisen DJ, Swedberg $\mathrm{K}$, Shi $\mathrm{H}$ et al (2011) Eplerenone in patients with systolic heart failure and mild symptoms. N Engl J Med 364(1):11-21

3. Gijsberts CM, Benson L, Dahlström U, Sim D, Yeo DPS, Ong HY et al (2016) Ethnic differences in the association of QRS duration with ejection fraction and outcome in heart failure. Heart (British Cardiac Society) 102:1464-1471

4. Emdin CA, Conrad N, Kiran A, Salimi-Khorshidi G, Woodward M, Anderson SG et al (2017) Variation in hospital performance for heart failure management in the National Heart Failure Audit for England and Wales. Heart 103(1):55-62

5. Khan MA, Satchithananda DK, Mamas MA (2016) The importance of interactions between atrial fibrillation and heart failure. Clin Med (Lond, Engl) 16(3):272-276

6. Mamas MA, Caldwell JC, Chacko S, Garratt CJ, Fath-Ordoubadi F, Neyses L (2009) A meta-analysis of the prognostic significance of atrial fibrillation in chronic heart failure. Eur J Heart Failure 11(7):676-683
7. Mountantonakis SE, Grau-Sepulveda MV, Bhatt DL, Hernandez AF, Peterson ED, Fonarow GC (2012) Presence of atrial fibrillation is independently associated with adverse outcomes in patients hospitalized with heart failure: an analysis of get with the guidelines-heart failure. Circ Heart Fail 5(2):191-201

8. Romero R, Gaytan JM, Aguirre A, Llorens P, Gil V, Herrero P et al (2018) The role of atrial fibrillation in the short-term outcomes of patients with acute heart failure. Clin Res Cardiol. https ://doi.org/10.1007/s00392-018-1389-x

9. Llorens P, Javaloyes P, Martin-Sanchez FJ, Jacob J, HerreroPuente P, Gil V et al (2018) Time trends in characteristics, clinical course, and outcomes of 13,791 patients with acute heart failure. Clin Res Cardiol 107(10):897-913

10. Filippatos G, Farmakis D, Metra M, Cotter G, Davison BA, Felker GM et al (2017) Serelaxin in acute heart failure patients with and without atrial fibrillation: a secondary analysis of the RELAXAHF trial. Clin Res Cardiol 106(6):444-456

11. Rivero-Ayerza M, Scholte Op Reimer W, Lenzen M, Theuns DA, Jordaens L, Komajda M et al (2008) New-onset atrial fibrillation is an independent predictor of in-hospital mortality in hospitalized heart failure patients: results of the EuroHeart Failure Survey. Eur Heart J 29(13):1618-1624

12. Pedersen OD, Sondergaard P, Nielsen T, Nielsen SJ, Nielsen ES, Falstie-Jensen N et al (2006) Atrial fibrillation, ischaemic heart disease, and the risk of death in patients with heart failure. Eur Heart J 27(23):2866-2870

13. Shoaib A, Farag M, Nolan J, Rigby A, Patwala A, Rashid M et al (2018) Mode of presentation and mortality amongst patients hospitalized with heart failure? A report from the first Euro heart failure survey. Clin Res Cardiol. https://doi.org/10.1007/s0039 2-018-1380-6

14. Miro O, Gil VI, Martin-Sanchez FJ, Jacob J, Herrero P, Alquezar A et al (2018) Short-term outcomes of heart failure patients with reduced and preserved ejection fraction after acute decompensation according to the final destination after emergency department care. Clin Res Cardiol 107(8):698-710

15. Meyer S, Teerlink JR, Metra M, Ponikowski P, Cotter G, Davison BA et al (2017) Sex differences in early dyspnea relief between men and women hospitalized for acute heart failure: insights from the RELAX-AHF study. Clin Res Cardiol 106(4):280-292

16. Cleland JG, McDonagh T, Rigby AS, Yassin A, Whittaker T, Dargie HJ (2011) The national heart failure audit for England and Wales 2008-2009. Heart 97(11):876-886

17. Cleland J, Dargie H, Hardman S, Mcdonagh T, Mitchell P (2013) National Heart Failure Audit April 2012-March 2013. http:// www.ucl.ac.uk/nicor/audits/heartfailure/additionalfiles/pdfs/annua lreports/NHFA13medium.pdf. Accessed 21 Nov 2013

18. Austin PC (2009) Balance diagnostics for comparing the distribution of baseline covariates between treatment groups in propensity-score matched samples. Stat Med 28(25):3083-3107

19. Vandenbroucke JP, von Elm E, Altman DG, Gotzsche PC, Mulrow CD, Pocock SJ et al (2007) Strengthening the reporting of observational studies in epidemiology (STROBE): explanation and elaboration. Epidemiology 18(6):805-835

20. Abualnaja S, Podder M, Hernandez AF, McMurray JJ, Starling RC, O'Connor CM et al (2015) Acute heart failure and atrial fibrillation: insights from the acute study of clinical effectiveness of nesiritide in decompensated heart failure (ASCEND-HF) trial. J Am Heart Assoc 4(8):e002092

21. McDonagh T, Cleland J, Dargie HJ, Whittaker T, Standing M, Mitchell M et al (2012) National Heart Failure Audit April 2010March 2011

22. Anter E, Jessup M, Callans DJ (2009) Atrial fibrillation and heart failure: treatment considerations for a dual epidemic. Circulation 119(18):2516-2525 
23. Caldwell J, Mamas M, Garratt C, Neyses L (2008) How common is asymptomatic paroxysmal atrial fibrillation in chronic heart failure? Scand Cardiovasc J SCJ 42(6):366 (author reply 7)

24. Kareti KR, Chiong JR, Hsu SS, Miller AB (2005) Congestive heart failure and atrial fibrillation: rhythm versus rate control. J Card Fail 11(3):164-172

25. Sramko M, Wichterle D, Melenovsky V, Franekova J, Clemens M, Fukunaga $M$ et al (2019) Independent effect of atrial fibrillation on natriuretic peptide release. Clin Res Cardiol 108(2):142-149

26. Llach A, Molina CE, Prat-Vidal C, Fernandes J, Casado V, Ciruela $F$ et al (2011) Abnormal calcium handling in atrial fibrillation is linked to up-regulation of adenosine A2A receptors. Eur Heart J 32(6):721-729

27. Li D, Fareh S, Leung TK, Nattel S (1999) Promotion of atrial fibrillation by heart failure in dogs. Atrial Remodel Differ Sort 100(1):87-95

28. Dries DL, Exner DV, Gersh BJ, Domanski MJ, Waclawiw MA, Stevenson LW (1998) Atrial fibrillation is associated with an increased risk for mortality and heart failure progression in patients with asymptomatic and symptomatic left ventricular systolic dysfunction: a retrospective analysis of the SOLVD trials. Studies of left ventricular dysfunction. J Am Coll Cardiol 32(3):695-703

29. Swedberg K, Olsson LG, Charlesworth A, Cleland J, Hanrath $\mathrm{P}$, Komajda $\mathrm{M}$ et al (2005) Prognostic relevance of atrial fibrillation in patients with chronic heart failure on long-term treatment with beta-blockers: results from COMET. Eur Heart J 26(13):1303-1308

30. Middlekauff HR, Stevenson WG, Stevenson LW (1991) Prognostic significance of atrial fibrillation in advanced heart failure. A study of 390 patients. Circulation 84(1):40-48

31. Linssen GC, Rienstra M, Jaarsma T, Voors AA, van Gelder IC, Hillege HL et al (2011) Clinical and prognostic effects of atrial fibrillation in heart failure patients with reduced and preserved left ventricular ejection fraction. Eur J Heart Fail 13(10):1111-1120

32. Kotecha D, Chudasama R, Lane DA, Kirchhof P, Lip GY (2016) Atrial fibrillation and heart failure due to reduced versus preserved ejection fraction: a systematic review and meta-analysis of death and adverse outcomes. Int J Cardiol 203:660-666

33. Kober L, Swedberg K, McMurray JJ, Pfeffer MA, Velazquez EJ, Diaz R et al (2006) Previously known and newly diagnosed atrial fibrillation: a major risk indicator after a myocardial infarction complicated by heart failure or left ventricular dysfunction. Eur J Heart Fail 8(6):591-598

34. Schneider MP, Hua TA, Böhm M, Wachtell K, Kjeldsen SE, Schmieder RE (2010) Prevention of atrial fibrillation by reninangiotensin system inhibition. A meta-analysis. J Am Coll Cardiol 55(21):2299-2307

35. Jibrini MB, Molnar J, Arora RR (2008) Prevention of atrial fibrillation by way of abrogation of the renin-angiotensin system: a systematic review and meta-analysis. Am J Ther 15(1):36-43

36. Goette A, Schon N, Kirchhof P, Breithardt G, Fetsch T, Hausler KG et al (2012) Angiotensin II-antagonist in paroxysmal atrial fibrillation (ANTIPAF) trial. Circ Arrhythm Electrophysio $5(1): 43-51$

37. Yusuf S, Healey JS, Pogue J, Chrolavicius S, Flather M, Hart RG et al (2011) Irbesartan in patients with atrial fibrillation. N Engl J Med 364(10):928-938

38. Kotecha D, Flather MD, Altman DG, Holmes J, Rosano G, Wikstrand J et al (2017) Heart rate, heart rhythm, and prognostic benefits of beta-blockers in heart failure: individual patient-data meta-analysis. J Am Coll Cardiol 69(24):2885-2896. https://doi. org/10.1016/j.jacc.2017.04.001

39. Mordi IR, Santema BT, Kloosterman M, Choy AM, Rienstra M, van Gelder I et al (2019) Prognostic significance of changes in heart rate following uptitration of beta-blockers in patients with sub-optimally treated heart failure with reduced ejection fraction in sinus rhythm versus atrial fibrillation. Clin Res Cardiol. https ://doi.org/10.1007/s00392-018-1409-x

40. Swedberg K, Zannad F, McMurray JJ, Krum H, van Veldhuisen DJ, Shi $\mathrm{H}$ et al (2012) Eplerenone and atrial fibrillation in mild systolic heart failure: results from the EMPHASIS-HF (Eplerenone in Mild Patients Hospitalization And SurvIval Study in Heart Failure) study. J Am Coll Cardiol 59(18):1598-1603

41. Farmakis D, Simitsis P, Bistola V, Triposkiadis F, Ikonomidis I, Katsanos S et al (2017) Acute heart failure with mid-range left ventricular ejection fraction: clinical profile, in-hospital management, and short-term outcome. Clin Res Cardiol 106(5):359-368

42. Ponikowski P, Voors AA, Anker SD, Bueno H, Cleland JG, Coats $\mathrm{AJ}$ et al (2016) 2016 ESC guidelines for the diagnosis and treatment of acute and chronic heart failure. Revista espanola de cardiologia (Engl ed) 69(12):1167

\section{Affiliations}

\section{Simon G. Anderson ${ }^{1,2,3}$. Ahmad Shoaib ${ }^{4}$. Phyo Kyaw Myint ${ }^{5}$. John G. Cleland ${ }^{6,7}$. Suzanna M. Hardman ${ }^{8}$. Theresa A. McDonagh ${ }^{9} \cdot$ Henry Dargie $^{10} \cdot$ Bernard Keavney $^{1} \cdot$ Clifford J. Garratt $^{1}$. Mamas A. Mamas ${ }^{4}$}

1 Division of Cardiovascular Sciences, Faculty of Biology, Medicine and Health, The University of Manchester, Manchester, UK

2 Department of Cardiology, North West Heart Centre, University Hospitals of South, Manchester, UK

3 The George Alleyne Chronic Disease Research Centre, Caribbean Institute for Health Research (CAIHR), The University of the West Indies, Bridgetown, Barbados

4 Keele Cardiovascular Research Group, Centre for Prognosis Research, Institute for Primary Care and Health Sciences, University of Keele and Royal Stoke Hospital, Stoke-on-Trent, UK

5 Institute of Applied Health Sciences, University of Aberdeen, Aberdeen, UK
6 Robertson Centre for Biostatistics and Clinical Trials, University of Glasgow, Glasgow, Scotland, UK

7 National Heart and Lung Institute, Imperial College, London, UK

8 Clinical and Academic Department of Cardiovascular Medicine, Whittington Hospital, London, UK

9 Faculty of Life Sciences and Medicine, King's College London, London, UK

10 Institute of Cardiovascular and Medical Sciences, University of Glasgow, Glasgow, UK 